01

\title{
Возможности дистрибутивных гауссовых $s$-функций для расчета энергии корреляции молекул в основном и возбужденных состояниях
}

\author{
(C) В.Н. Глушков \\ Днепровский национальный университет им. О. Гончара, \\ 49000 Днепр, Украина \\ e-mail: v_n_glushkov@yahoo.com
}

Поступила в редакцию 16.07.2020 г.

В окончательной редакции 07.10.2020 г.

Принята к публикации 14.10.2020 г.

Исследованы возможности дистрибутивных базисов из гауссовых $s p$-функций для расчета энергии корреляции молекул во втором порядке $\left(E^{(2)}\right)$ теории возмущений Меллера-Плессе (ТВМП). Эффективность таких базисов продемонстрирована сравнением величины $E^{(2)}$, полученной в дистрибутивных и стандартных атом-центрированных базисах. Расчеты проведены как для основного, так и возбужденного состояний с симметрией основного. Для этого развит аналог ТВМП для возбужденных состояний, который сохраняет преимущества ТВМП для основного состояния. Показано, что дистрибутивные $s p$-функции обеспечивают точность вычисления $E^{(2)}$, которая сравнима с точностью атом-центрированных базисов, включающих $s$-, $p$ и $d$-функции.

Ключевые слова: дистрибутивные функции, энергия корреляции, теория возмущений для возбужденных состояний.

DOI: $10.21883 / \mathrm{OS} .2021 .02 .50549 .196-20$

\section{1. Введение}

Известно, что теория электронной корреляции является одним из полезных инструментов для предсказания точных энергий в молекулярной физике и квантовой химии. Критерии построения и отбора молекулярных и атомных базисов для расчета энергии корреляции отличаются от таковых для энергии в приближении Хартри-Фока (ХФ). В настоящее время разработаны различные рецепты генерации базисов для молекул, которые строятся, как правило, из гауссовых функций, центрированных непосредственно на атомах (атомцентрированные (АЦ) базисы) [1-3] и для которых интенсивно исследуются вопросы базисной сходимости [4-6]. Эффекты поляризации электронного распределения в АЦ базисах описываются включением функций, отвечающих более высоким квантовым числам углового момента $l$, например, функций $p, d, f, g, h$ и т. д. Одним из важных моментов в построении таких базисов является концепция иерархии генерируемых функций, которая ведет к систематическому улучшению результатов от одного уровня в иерархической лестнице к другому. Примером таких базисов, в частности, являются популярные корреляционно-согласованные (correlation-consistent) базисы aug-cc-pVXZ [7-10], где $X$ соответствует значению $l(X=D: l=2, X=T: l=3$ и т.д.). И хотя подобные базисы включены практически во все пакеты современных квантово-химических программ, они к сожалению показывает слабую сходимость вычислений с увеличением размерности базиса. Так, например, для молекулы водорода предел ХФ достигается в достаточно большом базисе, состоящем из $22 s-, 13 p-, 8 d-, 4 f-$, $2 g$ - и 2h-функций [11]. Сходимость корреляционной энергии с увеличением базиса носит более медленный характер, чем сходимость энергии ХФ: даже очень большие базисные наборы, содержащие функции вплоть до $l=6$ дают только 95-98\% точной корреляционной энергии [12]. Существенное улучшение сходимости в расчетах энергии корреляции может быть достигнуто на пути применения так называемых явно коррелированных методов, т.е. методов, которые используют функции, явно зависящие от межэлектронного расстояния $r_{12}$. Впервые это было сделано Хиллераасом на примере атома Не в работе [13], где была продемонстрирована эффективность такого подхода. В дальнейшем эта идея получила обобщение и была реализована в рамках как метода конфигурационного взаимодействия (CI-R12 method) [14-17], так и теории возмущений Меллера-Плессе (ТВМП) [18] второго порядка (MP2R12 method) [19]. Пока такие методы не стали рутинными в квантово-механических расчетах, так как они требуют значительных компьютерных затрат, связанных со сложными многоэлектронными интегралами. Однако соответствующие результаты составляют превосходную эталонную базу для сравнения с результатами других приближенных методов, чтобы оценить их эффективность.

Наряду с введением в базис функций с большим значением $l$ поляризационные эффекты могут быть также описаны применением дистрибутивных базисов 
(те. центровки соответствующих гауссовых функций распределены вдоль молекулярной оси). Основным преимуществом дистрибутивных базисов является их компактность и простота вычислений, так как можно ограничиться функциями с меньшим квантовым числом углового момента. Поэтому интерес к моделированию таких базисов в настоящее время не уменьшается [20]. Дистрибутивные базисы применялись в вариационных расчетах колебательной структуры молекул $[21,22]$ и реже используются при исследовании электронной структуры молекул. Основная проблема построения таких базисов - определение мест центровки гауссовых функций $\mathbf{R}_{p} \Rightarrow\left\{X_{p}, Y_{p}, Z_{p}\right\}$. В расчетах поляризуемости полезной оказалась модель распределения функций, предложенная A. Sadlej [23]. Проблема определения электронной энергии наиболее полно обсуждалась в работах С. Уилсона с сотрудниками, где были предложены полезные полуэмпирические схемы генерации дистрибутивных $s$-базисов для расчета энергии основного состояния [24-26]. На примере простых молекул было продемонстрировано, что базисные наборы из дистрибутивных $s$-функций способны в приближении ХФ обеспечивать точность расчета энергии на уровне микрохартри ( $\mu$ Hartree) [27-30]. Однако систематические исследования возможностей дистрибутивных функций для расчета энергии корреляции основного состояния практически не проводились, за исключением работы [31] и ссылок в ней, где рассматривались полуэмпирические модели генерации $s$-функций для расчета корреляционной энергии основного состояния молекулы водорода во втором порядке теории возмущений. Для возбужденных состояний подобные исследования нам не известны.

В настоящей работе исследуется эффективность дистрибутивных функций $s p$-типа для расчета энергии электронной корреляции в основном и возбужденных состояниях. В частности, оценивается точность, с которой энергия корреляции может быть рассчитана во втором порядке ТВМП (МР2). Анализ результатов проводится на примере простых молекул в основном и возбужденных состояниях с симметрией основного. Сложность задачи о нахождении центровок функций приводит к пониманию, что, видимо, не существует единой стратегии в построении дистрибутивных базисов. Поэтому на данном этапе исследования мы применяли вариационные методы (Релея-Ритца и Хиллерааса), где как параметры орбитальных экспонент $\xi_{p}$, так и центровки $\mathbf{R}_{p}$ определялись минимизацией соответствующего функционала.

В следующем разделе кратко обсуждаются уравнения ХФ и поправки второго порядка к энергии только возбужденного состояния, так как соответствующие формулы для основного состояния хорошо известны [32]. Раздел 3 демонстрирует возможности дистрибутивных $s p$-базисов для определения корреляционной энергии молекул $\mathrm{H}_{2}$ и $\mathrm{LiH}$ в основном состоянии (разд. 3.1). Для этого проводится сравнение МР2-энергий, полученных в дистрибутивном и стандартных базисах aug-cc-pVXZ, а также с высокоточными результатами. Особенности расчета энергии корреляции в приближении МР2 для возбужденных состояний с симметрией основного рассматриваются в разд. 3.2 на примере молекулы с открытыми электронными оболочками, НеН.

\section{2. Теория возмущений для возбужденных состояний на основе одного опорного детерминанта Слэтера}

В рамках формализма волновой функции можно выделить следующие подходы для расчетов возбужденных состояний систем с открытыми оболочками:

- методы конфигурационного взаимодействия (КВ),

- формализм теории связанных кластеров,

- техника на основе уравнений движения,

- многоопорная (multi-reference) теория возмущений.

Используя расширенные стандартные базисные наборы из $s$-, $p$-, $d-, f-, g$ - и $h$-функций, эти подходы способны обеспечить целевую точность энергии возбужденных состояний простых систем. Как отмечалось во введении, соответствующие результаты составляют эталонную базу, чтобы оценить эффективность других новых методов. Для основного состояния популярным инструментом для учета корреляционных эффектов стала ТВМП, которая строится на одном опорном детерминанте Слэтера. Популярность такой техники стала возможной благодаря сочетанию эффективности приближения ХФ и простоты расчета низших порядков ТВМП. Реализация подобного подхода для возбужденных состояний, имеющих ту же симметрию, что и основное, наталкивается на трудности построения опорного детерминанта, который удовлетворяет условию ортогональности состояний. Одна из попыток решения этой задачи была предложена в работах [33,34]. Впоследствии результаты этих работ были развиты для многократно возбужденных состояний [35]. Следует отметить, что были также предложены методы самосогласованного поля [36] , которые явно не требуют ортогональности. Возможность построения теории возмущения для возбужденных состояний в указанных работах не рассматривалась.

Ниже мы приводим лишь основные уравнения, определяющие орбитали возбужденного состояния в приближении ХФ и выражение для поправки второго порядка ТВМП для энергии (детали см. в [33,34]). Для простоты ограничимся первым возбужденным состоянием $\Phi$, построенным на орбиталях $\left\{\varphi_{i}\right\}, i=1,2, \ldots, n$, которые определяются модифицированными уравнениями Хартри-Фока:

$$
\begin{aligned}
& P\left(F^{\alpha}-\lambda_{s} P^{\beta}+\lambda_{o} P_{n}^{\alpha}-\varepsilon_{i}^{\alpha}\right) P\left|\varphi_{i}^{\alpha}\right\rangle=0, \lambda_{s}, \lambda_{o} \rightarrow \infty, \\
& P\left(F^{\beta}+\lambda_{s} Q^{\alpha}+\lambda_{o} P_{n}^{\alpha}-\varepsilon_{i}^{\beta}\right) P\left|\varphi_{i}^{\beta}\right\rangle=0, \quad i=1,2, \ldots, M .
\end{aligned}
$$


В уравнениях (1) введены следующие обозначения: $F^{\alpha}$ и $F^{\beta}-$ стандартные фокианы для спина $\alpha$ и $\beta$ в теории неограниченного метода ХФ; $P$ - ортопроектор на подпространство, которое определяется выбранным базисом с размерностью $M>n$. В нашем случае предусмотрено, что подпространства для возбужденного и основного состояний могут отличаться; $P^{\beta}, Q^{\alpha}, P_{n}^{\alpha}-$ ортопроекторы на подпространство занятых орбиталей $\beta$-роя, на подпространство виртуальных орбиталей $\alpha$-роя и $P_{n}^{\alpha}=\left|\varphi_{0 n}^{\alpha}\right\rangle\left\langle\varphi_{0 n}^{\alpha}\right|-$ ортопроектор на направление, которое определяется занятой орбиталью основного состояния $\varphi_{0 n}^{\alpha}$ с соответствующей максимальной орбитальной энергией $\varepsilon_{0 n}^{\alpha}$. Отметим, что уравнения (1) определяют пространственную часть спин-орбиталей. Поэтому в общем случае $P^{\beta}\left|\varphi_{i}^{\alpha}\right\rangle \neq 0$.

В теории метода асимптотического проектирования (АП), предложенного ранее [33,37], показано, что в пределе $\lambda_{s}, \lambda_{o} \rightarrow \infty$ эти множители обеспечивают спиновую чистоту $\left(\lambda_{s} \rightarrow \infty\right)$ и ортогональность состояний $\left(\lambda_{o} \rightarrow \infty\right)$. Полезно отметить, что в случае основного состояния $\left(\lambda_{o}=0\right)$ орбитали уравнения (1) приводят к энергии, идентичной методу Рутана для открытых оболочек. Однако, как известно, уравнения Рутана являются уравнениями 5-й или 7-й степени относительно коэффициентов разложения орбиталей по базисным функциям, тогда как уравнения (1) являются кубическими для этих коэффициентов. В практических расчетах значения $\lambda_{s}=100$ a.u. и $\lambda_{o}=10000$ a.u. обеспечивали требуемую точность.

Как видно, структура уравнений (1) напоминает структуру уравнений неограниченного метода ХФ $\left(\lambda_{o}=0\right.$, $\left.\lambda_{s}=0\right)$. Это позволило построить аналог ТВМП для возбужденного состояния. Так, например, поправка второго порядка к энергии имеет вид [34]

$$
\begin{aligned}
E^{(2)}= & \sum_{i>j}^{\text {occ }} \sum_{a>b}^{\text {virt }} \frac{\left|\left(\varphi_{a} \varphi_{i} \mid \varphi_{b} \varphi_{j}\right)-\left(\varphi_{a} \varphi_{j} \mid \varphi_{b} \varphi_{i}\right)\right|^{2}}{\varepsilon_{i}+\varepsilon_{j}-\varepsilon_{a}-\varepsilon_{b}} \\
& -\left\langle\Phi|H| \Phi_{0}\right\rangle\left\langle\Phi_{0}^{(1)} \mid \Phi\right\rangle .
\end{aligned}
$$

Суммирование в (2) включает также суммирование по спину. Индексы $i, j$ используются для занятых орбиталей, тогда как $a, b$ для виртуальных. $\Phi_{0}$ и $\Phi_{0}^{(1)}-$ однодетерминантная волновая функция основного состояния и поправка первого порядка к ней соответственно, $H$ - гамильтониан системы. Первое слагаемое в (2) по форме совпадает с поправкой второго порядка к энергии основного состояния. Появление второго слагаемого обусловлено учетом ортогональности $\left\langle\Phi_{0} \mid \Phi\right\rangle=0$. В общем случае связывающий элемент $\left\langle\Phi|H| \Phi_{0}\right\rangle$ не равен нулю, так как теорема Бриллюэна не выполняется. В работе [38] на примере простых систем было показано, что $\left\langle\Phi|H| \Phi_{0}\right\rangle \ll 1$, если $\left\langle\Phi_{0} \mid \Phi\right\rangle \sim 10^{-5}$. Так как $\left\langle\Phi_{0}^{(1)} \mid \Phi\right\rangle<1$, то на данном этапе расчетов мы пренебрегали вторым слагаемым. Таким образом, для возбужденного состояния вычислительные затраты сравнимы с затратами для основного.

\section{3. Обсуждение результатов расчета $E^{(2)}$ для основного и возбужденного состояний}

В данном разделе рассматриваются возможности дистрибутивных гауссовых функций $s p$-типа путем вычисления и сравнения поправок второго порядка к энергии $E^{(2)}$ основного состояния молекул $\mathrm{H}_{2}$ и $\mathrm{LiH}$ и соответствующие поправки для молекулы НеН в первом возбужденном состоянии $A^{2} \Sigma^{+}$. Для оценки точности проводится сравнение с результатами $E^{(2)}$, полученными в стандарных базисах и методом MP2-R12 ( $\mathrm{H}_{2}$ и LiH), а также с результатами метода КВ $(\mathrm{HeH})$. Для исследуемых молекул, ось которых расположена вдоль оси $Z$, в данной работе были использованы следующие наборы дистрибутивных гауссовых базисных функций: $s$-типа

$$
\begin{gathered}
\chi_{m^{s}}(x, y, z)=\exp \left\{-\zeta_{m^{s}}\left[x^{2}+y^{2}+\left(z-Z_{m^{s}}\right)^{2}\right]\right\}, \\
m^{s}=1,2, \ldots, M^{s},
\end{gathered}
$$

$p$-типа $\left(p_{x}\right.$ и $\left.p_{y}\right)$

$$
\begin{gathered}
\chi_{m^{p_{x}}}=x \exp \left\{-\zeta_{m^{p_{x}}}\left[x^{2}+y^{2}+\left(z-Z_{m^{p_{x}}}\right)^{2}\right]\right\}, \\
m^{p_{x}}=1,2, \ldots, M^{p x}, \\
\chi_{m^{p_{y}}}=y \exp \left\{-\zeta_{m^{p_{y}}}\left[x^{2}+y^{2}+\left(z-Z_{m^{p_{y}}}\right)^{2}\right]\right\}, \\
m^{p_{y}}=1,2, \ldots, M^{p y} .
\end{gathered}
$$

Здесь $\xi_{m}$ и $Z_{m}$ - параметры экспонент и центровки, которые определяются минимизацией соответствующего функционала. $M^{s}, M^{p x}$ и $M^{p y}$ - число $s$-, $p_{x}$ - и $p_{y}$ функций соответственно. При этом ввиду симметрии $\xi_{m^{p_{x}}}=\xi_{m^{p_{y}}}, Z_{m^{p_{x}}}=Z_{m^{p_{y}}}$ и $M^{p x}=M^{p y}$. Следует отметить, что в стандартных АЦ базисах $p$-орбиталь подразумевает три функции - $p_{x}, p_{y}$ и $p_{z}$. В нашем методе используются только две функции $p_{x}$ и $p_{y}$, так как поведение $p_{z}$ имитируется распределением $s$-функций вдоль молекулярной оси $O Z$. Этот факт существенно упрощает вычисление необходимых матричных элементов, для которых были использованы результаты работы [39]

\section{1. Дистрибутивные $s$-базисы и MP2-энергия корреляции для $\mathrm{H}_{2}$ и $\mathrm{LiH}$}

Наши расчеты энергии ХФ $E_{\mathrm{HF}}$ и поправки второго порядка $E^{(2)}$ и $E_{\mathrm{HF}}+E^{(2)}$ для молекулы $\mathrm{H}_{2}$ были проведены для межъядерного расстояния $R=1.400 \mathrm{bohr}$. Peзультаты, полученные для последовательности дистрибутивных увеличивающихся базисов типа $2 x 15 s+2 x 12 s+$ $+4 k\left(p_{x}+p_{y}\right), \quad k=1,2,3,4,5,6,7$, а также энергии, полученные с применением стандартных базисов [12], 
Таблица 1. Полная $\left(E=E^{\mathrm{HF}}+E^{(2)}\right)$ (Hartree) и корреляционная $E^{(2)}$ (mHartree) энергии основного состояния молекулы $\mathrm{H}_{2}$, вычисленные в дистрибутивных $s p$-базисах типа $30 s+24 s+4 k\left(p_{x}+p_{y}\right), k=1,2,3,4,5,6,7$, и стандартных базисах различной размерности $\left(E^{\mathrm{HF}}(30 s)=-1.13362933\right.$ Hartree, $\left.R=1.4 \mathrm{bohr}\right)$

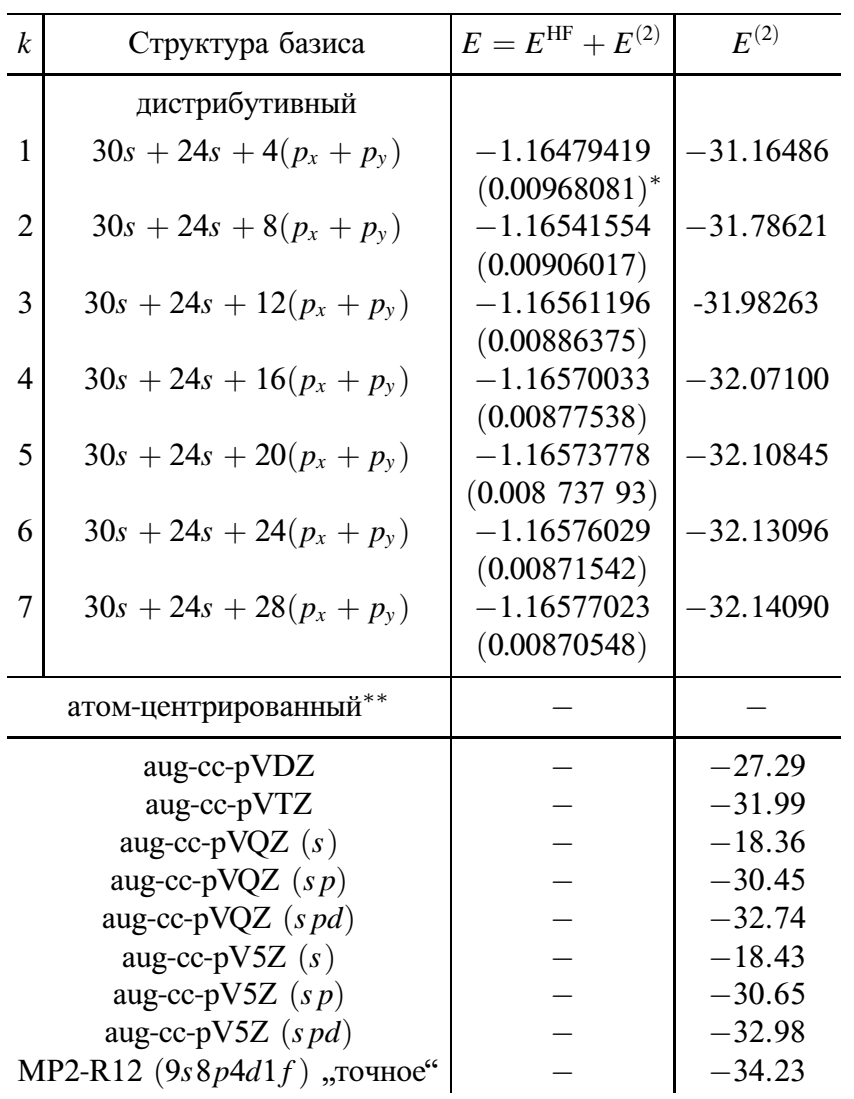

Примечание. * В скобках указано отклонение от „точного значения: $E-E^{\text {exact }}\left(\right.$ Hartree), $E^{\text {exact }}=-1.17447571$ Hartree $[15] .{ }^{* *}$ Данные из [12].

представлены в табл. 1. Обозначения базисов учитывают симметрию и подразумевают, что вычисления проводились в несколько этапов. На первом этапе параметры экспонент $\xi_{m}$ и центровки $Z_{m}$ базиса, состоящего из $30 s=2 \times 15 s$ функций, определялись минимизацией ХФ энергии. Такой базис обеспечивает точность на ХФ-уровне $\sim 0.3 \mu$ Hartree от „точного“ значения, т.е. $E_{\mathrm{HF}}-E_{\mathrm{HF}}^{\text {exact }}$ $=-1.13362933-1.13362957=0.00000024$ Hartree. „Точное“ значение $E_{\mathrm{HF}}^{\text {exact }}=-1.13362957$ Hartree получено численным методом конечных разностей [40]. Понятно, что дальнейшее расширение базиса только $s$-функциями практически не улучшит $E_{\mathrm{HF}}$. Поэтому дополнительные $s$-функции $24 s=2 \times 12 s$ были выбраны так, что их параметры определялись минимизацией функционала Хиллерааса, т.е. максимального понижения $E^{(2)}$ при фиксированных $30 s$-функциях. На заключительной стадии параметры p-функций также были найдены из условия минимума функционала Хиллерааса.
Таблица 2. Полная $\left(E=E^{\mathrm{HF}}+E^{(2)}\right)$ (Hartree) и корреляционная $E^{(2)}$ (mHartree) энергии основного состояния молекулы $\mathrm{LiH}$, вычисленные в дистрибутивных базисах $s p$-типа $36 s+3 k\left(p_{x}+p_{y}\right), k=0,1,2,3,4,5,6$, и в стандартных базисах различной размерности (энергия в приближении ХФ: $E^{\mathrm{HF}}=-7.987329$ Hartree, $R=3.015 \mathrm{bohr}$ )

\begin{tabular}{c|c|c|c|c}
\hline$k$ & Структура базиса & $E^{\mathrm{HF}}+E^{(2)}$ & $E^{(2)}$ & $\Delta^{*}$ \\
\hline & дистрибутивный & & & \\
0 & $36 s$ & -8.024433 & -37.104 & - \\
1 & $36 s+3\left(p_{x}+p_{y}\right)$ & -8.045800 & -58.451 & 21.347 \\
2 & $36 s+6\left(p_{x}+p_{y}\right)$ & -8.049595 & -62.266 & 3.815 \\
3 & $36 s+9\left(p_{x}+p_{y}\right)$ & -8.051514 & -64.185 & 1.919 \\
4 & $36 s+12\left(p_{x}+p_{y}\right)$ & -8.052433 & -65.104 & 0.855 \\
5 & $36 s+15\left(p_{x}+p_{y}\right)$ & -8.052818 & -65.489 & 0.385 \\
6 & $36 s+18\left(p_{x}+p_{y}\right)$ & -8.053025 & -65.696 & 0.207 \\
\hline \multicolumn{2}{|c|}{ атом-центрированный } & & & \\
$(14 s 9 p, 8 s 4 p)^{* *}$ & - & -61.78 & - \\
$(14 s 9 p 4 d, 8 s 4 p)$ & - & -63.06 & - \\
$(14 s 9 p 4 d 3 f, 8 s 4 p)$ & - & -63.68 & - \\
$(14 s 9 p 4 d, 8 s 4 p 3 d)$ & - & -65.24 & - \\
$(14 s 9 p 4 d 3 f, 8 s 4 p 3 d)$ & - & -65.40 & - \\
МР2-R $12-$, Точное & & & \\
$(11 s 7 p 4 d 1 f, 9 s 6 p 3 d 1 f)$ & - & -72.76 & -
\end{tabular}

Примечание. ${ }^{*} \Delta=E_{k-1}^{(2)}-E_{k}^{(2)}$ (mHartree). ** Данные из [12].

На наш взляд, сравнение результатов будет корректным, если его проводить между значениями, полученными в базисах с одинаковым типом функций. В нашем случае это $s p$-функции как для дистрибутивного, так и стандартных базисов (aug-ccpVQZ(sp), aug-cc-pV5Z(sp)). Это дает для дистрибутивного $E^{(2)}=-32.14 \mathrm{mHartree} \quad$ и для стандартных $E^{(2)}=-30.45$ и -30.65 mHartree соответственно (табл. 1). Более того, наш $s p$-результат сравним с результатами $s p d$-базиса aug-cc-pVQZ(spd), $\left(E^{(2)}=-32.74\right.$ mHartree). При этом он составляет $\sim 94 \%$ от „точной“ МР2 корреляционной энергии (-34.24 mHartree [12]).

В табл. 2 представлены полные и МР2-корреляционные энергии для LiH при межъядерном расстоянии $R=3.015 \mathrm{bohr}$, полученные в дистрибутивных и стандартных АЦ базисах. Наши результаты получены в базисах, состоящих из $s$ - и $p$-функций. Структуру базисов мы обозначили как $36 s+3 k\left(p_{x}+p_{y}\right)$, $k=0,1,2,3,4,5,6$. Алгоритм вычислений напоминает порядок выполнения расчетов для $\mathrm{H}_{2}$. Сначала была рассчитана $E^{\mathrm{HF}}=-7.987329$ Hartree, что сравнимо со значением, полученным методом конечных разностей [40] $E^{\text {exact }}=-7.987352$ Hartree. После этого, добавляя $p$-функции, распределенные вдоль молекулярной оси, была вычислена полная и МР2-корреляционная энергии. Чтобы проиллюстрировать, как распределяются центры функций, в табл. 3 приведены параметры экспонент и центровки функции базиса $36 s+18\left(p_{x}+p_{y}\right)$. 
Таблица 3. Параметры экспонент $\xi_{m}$ и центровки $Z_{m}$ базисных функций $s$ - и $p$-типов для молекулы LiH с расстоянием $R_{\mathrm{LiH}}=3.015$ bohr (базис $36 s+18\left(p_{x}+p_{y}\right)$, начало координат связано с атомом Li)

\begin{tabular}{|c|c|c|c|c|}
\hline$m$ & $\zeta_{m s}$ & $Z_{m s}$ & $\xi_{m p}$ & $Z_{m p}$ \\
\hline 1 & $0.9793009 \mathrm{E}+05^{*}$ & $-.3818457 E-05$ & $0.8937694 \mathrm{E}+00$ & $0.2874313 \mathrm{E}+01$ \\
\hline 2 & $0.1435563 \mathrm{E}+05$ & $0.1836716 \mathrm{E}-04$ & $0.4877177 \mathrm{E}+01$ & $0.8920844 \mathrm{E}-01$ \\
\hline 3 & $0.3208002 \mathrm{E}+04$ & $-.6025636 E-04$ & $0.1643354 \mathrm{E}+01$ & $-.1346144 E-01$ \\
\hline 4 & $0.1072106 \mathrm{E}+03$ & $0.6113779 \mathrm{E}-03$ & $0.4856972 \mathrm{E}+01$ & $0.2240790 \mathrm{E}-01$ \\
\hline 5 & $0.4410803 \mathrm{E}+02$ & $-.1240514 E-02$ & $0.5281247 \mathrm{E}+01$ & $-.1542645 E+00$ \\
\hline 6 & $0.1005598 \mathrm{E}+02$ & $0.1270273 \mathrm{E}-02$ & $0.1325357 \mathrm{E}+02$ & $0.2736026 \mathrm{E}-01$ \\
\hline 7 & $0.2058632 \mathrm{E}+02$ & $-.1308329 E-02$ & $0.1348440 \mathrm{E}+02$ & $-.2683119 E-01$ \\
\hline 8 & $0.2017287 \mathrm{E}+01$ & $-.3187405 E-02$ & $0.3441265 \mathrm{E}+00$ & $0.2995967 \mathrm{E}+01$ \\
\hline 9 & $0.8531669 \mathrm{E}+00$ & $0.4992078 \mathrm{E}-02$ & $0.9361676 \mathrm{E}+00$ & $0.3187920 \mathrm{E}+01$ \\
\hline 10 & $0.3947928 \mathrm{E}+00$ & $-.6374175 E-01$ & $0.2656881 \mathrm{E}+01$ & $0.2941006 \mathrm{E}+01$ \\
\hline 11 & $0.1175909 \mathrm{E}+00$ & $0.1025111 \mathrm{E}-01$ & $0.3435698 \mathrm{E}+00$ & $0.2142970 \mathrm{E}+01$ \\
\hline 12 & $0.4214455 \mathrm{E}-01$ & $0.2156079 \mathrm{E}+01$ & $0.9176827 \mathrm{E}+00$ & $0.2589148 \mathrm{E}+01$ \\
\hline 13 & $0.2722370 \mathrm{E}+03$ & $0.3014927 \mathrm{E}+01$ & $0.1038270 \mathrm{E}+01$ & $0.1344137 \mathrm{E}+01$ \\
\hline 14 & $0.1095051 \mathrm{E}+01$ & $0.2370044 \mathrm{E}+01$ & $0.1213567 \mathrm{E}+00$ & $0.2616406 \mathrm{E}+01$ \\
\hline 15 & $0.8877016 \mathrm{E}+00$ & $0.2996218 \mathrm{E}+01$ & $0.3506652 \mathrm{E}+02$ & $0.4108821 \mathrm{E}-02$ \\
\hline 16 & $0.3112971 \mathrm{E}+00$ & $0.2956645 \mathrm{E}+01$ & $0.3933298 \mathrm{E}+00$ & $0.1067074 \mathrm{E}+01$ \\
\hline 17 & $0.1122010 \mathrm{E}+00$ & $0.2873160 \mathrm{E}+01$ & $0.1212005 \mathrm{E}+00$ & $0.2339243 \mathrm{E}+01$ \\
\hline 18 & $0.1208391 E+00$ & $0.4311712 \mathrm{E}+00$ & $0.1683606 \mathrm{E}+01$ & $-.9372256 E-01$ \\
\hline 19 & $0.2925108 \mathrm{E}+03$ & $-.3453470 E-03$ & - & - \\
\hline 20 & $0.4947913 \mathrm{E}+01$ & $0.2002369 \mathrm{E}-01$ & - & - \\
\hline 21 & $0.8102012 \mathrm{E}+00$ & $0.2937500 \mathrm{E}-01$ & - & - \\
\hline 22 & $0.9396845 \mathrm{E}+01$ & $0.3012925 \mathrm{E}+01$ & - & - \\
\hline 23 & $0.2698038 \mathrm{E}+01$ & $0.3006632 \mathrm{E}+01$ & - & - \\
\hline 24 & $0.2631646 \mathrm{E}+00$ & $0.7138316 \mathrm{E}+00$ & - & - \\
\hline 25 & $0.1399783 \mathrm{E}+00$ & $0.4304292 \mathrm{E}+00$ & - & - \\
\hline 26 & $0.8983263 \mathrm{E}+03$ & $0.1446721 \mathrm{E}-03$ & - & - \\
\hline 27 & $0.4716241 \mathrm{E}+01$ & $0.5524010 \mathrm{E}-02$ & - & - \\
\hline 28 & $0.4092772 \mathrm{E}+02$ & $0.3014513 \mathrm{E}+01$ & - & - \\
\hline 29 & $0.6934428 \mathrm{E}+00$ & $0.1620101 \mathrm{E}+01$ & - & - \\
\hline 30 & $0.1555925 \mathrm{E}+01$ & $0.2060374 \mathrm{E}+00$ & - & - \\
\hline 31 & $0.1093181 \mathrm{E}+01$ & $0.2382497 \mathrm{E}+01$ & - & - \\
\hline 32 & $0.7243736 \mathrm{E}+00$ & $0.3495039 \mathrm{E}+01$ & - & - \\
\hline 33 & $0.2750338 \mathrm{E}+00$ & $0.2848336 \mathrm{E}+01$ & - & - \\
\hline 34 & $0.3068566 \mathrm{E}+00$ & $0.2950819 \mathrm{E}+01$ & - & - \\
\hline 35 & $0.6516569 \mathrm{E}+00$ & $0.1790597 \mathrm{E}+01$ & - & - \\
\hline 36 & $0.3020465 \mathrm{E}+00$ & $0.4158601 \mathrm{E}+01$ & - & - \\
\hline
\end{tabular}

Примечание. ${ }^{*} 0.9793009 E+05=0.9793009 \cdot 10^{5}$.

В 4-й колонке табл. 2 приведены энергетические разности $\Delta=E_{k-1}^{(2)}-E_{k}^{(2)}$, позволяющие оценить сходимость значений корреляционной энергии с увеличением базиса. Эффективность дистрибутивных базисов можно видеть, сравнивая $E^{(2)}$, полученную в данной работе и вычисленную с использованием стандартных атом-центрированных базисов [12] (нижняя часть табл. 2). Так, например, наши результаты в базисах $36 s+15\left(p_{x}+p_{y}\right)$ и $36 s+18\left(p_{x}+p_{y}\right)$ превосходят результаты стандартных базисов, включающих $s$-, $p$-, $d$ и $f$-функции. Хотя следует отметить, что сравнивая $E^{(2)}$ с высокоточными данными, полученными методом МР2$\mathrm{R} 12[12]\left(E^{(2)}=-72.76 \mathrm{mHartree}\right)$, можно сказать, что данная модель базиса (как и стандартная) не позволяет в дальнейшем существенно улучшить результаты. Наше наилучшее значение -65.696 mHartree покрыва- ет (как и стандартные базисы) 90\% от MP2-R12корреляционной энергии. Улучшение результатов можно ожидать, включая в базис дистрибутивные функции, центровка которых расположена вне молекулярной оси.

\section{2. Корреляционная энергия $\mathrm{HeH}$ в возбужденном состоянии $A^{2} \Sigma^{+}$}

В этом разделе кратко рассматриваются особенности расчета МР2-энергии корреляции молекулы НеН в первом возбужденном состоянии, которое имеет те же пространственную и спиновую симметрии, что и основное состояние $X^{2} \Sigma^{+}$с электронной конфигурацией $1 \sigma^{2} 2 \sigma$. Как известно, основное состояние НеН является несвязанным, тогда как потенциальная кривая возбуж- 
Таблица 4. Сходимость значений корреляционной энергии $E^{(2)}$ (mHartree) возбужденного состояния $A^{2} \Sigma^{+}$молекулы НеН для межъядерного расстояния $R=1.5 \mathrm{bohr}$, энергия в приближении ХФ: $E^{\mathrm{HF}}=-3.066710$ Hartree, базис $36 s+3 k\left(p_{x}+p_{y}\right)$, $k=0,1,2,3,4,5,6$

\begin{tabular}{c|c|c|c|c}
\hline$k$ & Структура базиса & $E_{\mathrm{HF}}+E^{(2)}$, Hartree & $E^{(2)}$ & $\Delta^{*}$ \\
\hline 0 & $36 s$ & -3.083996 & -17.283 & - \\
1 & $36 s+3\left(p_{x}+p_{y}\right)$ & -3.096147 & -29.437 & 12.154 \\
2 & $36 s+6\left(p_{x}+p_{y}\right)$ & -3.097370 & -30.657 & 1.220 \\
3 & $36 s+9\left(p_{x}+p_{y}\right)$ & -3.098063 & -31.353 & 0.696 \\
4 & $36 s+12\left(p_{x}+p_{y}\right)$ & -3.098201 & -31.491 & 0.138 \\
5 & $36 s+15\left(p_{x}+p_{y}\right)$ & -3.098309 & -31.579 & 0.088 \\
6 & $36 s+18\left(p_{x}+p_{y}\right)$ & -3.098320 & -31.610 & 0.031
\end{tabular}

Примечание. ${ }^{*} \Delta=E_{k-1}^{(2)}-E_{k}^{(2)}$ (mHartree).

денного состояния $A^{2} \Sigma^{+}$показывает минимум вблизи $R=1.5$ bohr. Поэтому все расчеты были выполнены для этого межъядерного расстояния.

На первом шаге была рассчитана энергия основного состояния в ХФ приближении, используя базис из $36 s$-функций, $E^{\mathrm{HF}}=-3.220302$ Hartree. Это значение хорошо согласуется с „точным“ результатом $E^{\text {exact }}=-3.220315$ Hartree, полученным методом конечных разностей. Следует отметить, что вычисления проводились для базисов различной размерности, как и в разд. 3.1. В работе мы приводим такую размерность $M^{s}$, которая обеспечивает точность для ХФ энергии молекул $\mathrm{LiH}$ и $\mathrm{HeH} \sim 20 \mu$ Hartree, а для $\mathrm{H}_{2} \sim 0.3 \mu$ Hartree. Следующий шаг - представление проектора $P_{n}^{\alpha}$ в виде $P_{n}^{\alpha}=|2 \sigma\rangle\langle 2 \sigma|$ и решение уравнений (1), чтобы определить орбитали возбужденного состояния $\left\{\varphi_{i}\right\}, i=1,2, \ldots, n$. В результате получаем энергию возбужденного состояния в приближении ХФ: $E^{\mathrm{HF}}=-3.066710$ Hartree. К сожалению, мы не можем провести корректное сравнение с другими авторами, так как в литературе отсутствуют расчеты энергии возбужденного состояния в приближении ХФ и энергии корреляции. Поэтому мы сравниваем наши полные энергии $E^{\mathrm{HF}}+E^{(2)}$ с высокоточными результатами, полученными в расширенном базисе методом КВ в работах $[41,42]$ : $E^{\text {exact }}=-3.113546$ Hartree.

Табл. 4 демонстрирует возможности дистрибутивных базисов, обозначенных как $36 s+3 k\left(p_{x}+p_{y}\right)$, $k=0,1,2,3,4,5,6$, для вычисления энергии корреляции молекулы НеН в возбужденном состоянии $A^{2} \Sigma^{+}$. Нетрудно видеть, что значение полной энергии в дистрибутивном базисе $36 s+18\left(p_{x}+p_{y}\right)$ : $E=E^{\mathrm{HF}}+E^{(2)}=-3.098320$ Hartree хорошо согласуется с $E^{\text {exact }}=-3.113546$ Hartree метода КВ $[41,42]$, который использует АЦ базисы и составляет $~ 99 \%$ энергии КВ.

В заключение отметим, что дистрибутивные базисы не следует рассматривать как альтернативу АЦ базисам. Напротив, их сочетание может оказаться полезным для повышения эффективности расчетов. Например, для орбиталей, описывающих область вблизи ядер, естественно использовать АЦ базисы, тогда как для внешней части более эффективными могут оказаться дистрибутивные функции. Такой комбинированный подход позволит улучшить сходимость вычислений корреляционной энергии с увеличением размерности базиса и уменьшит опасность возникновения приближенной линейной зависимости между функциями базиса. Улучшение результатов и их распространение на более сложные молекулы возможно на пути включения дистрибутивных функций с центрами вне молекулярной оси [20].

\section{Благодарности}

Автор благодарен проф. С. Уилсон (S. Wilson) за стимулирующие дискуссии.

\section{Конфликт интересов}

Автор заявляет, что у него нет конфликта интересов.

\section{Список литературы}

[1] Feller D., Davidson E.R. // Chem.Rev. 1986. V. 86. P. 681.

[2] Gill P.M.W. // Adv. Quant. Chem. 1994. V. 25. P. 141.

[3] Helgaker T., Taylor P.R. // In: Electronic Structure Theory. Part 2. Ed. Yarkony D., World Scientific, 1995. P. 727.

[4] Peterson G.A., Braunstein M. // J. Chem. Phys. 1985. V. 83. P. 5129.

[5] Feller D. // J. Chem. Phys. 1993. V. 98. P. 7059.

[6] Martin J.M.L., Taylor P.R. // J. Chem. Phys. 1997. V. 106. P. 8620.

[7] Helgaker T., Klopper W., Koch H., Noga J. // J. Chem. Phys. 1997. V. 106. P. 9639.

[8] Dunning T.H., Jr. // J. Chem. Phys. 1989. V. 90. P. 1007.

[9] Woon D.E., Dunning T.H., Jr. // J. Chem. Phys. 1994. V. 100. P. 2975.

[10] Wilson A.K., van Mourik T., Dunning T.H., Jr. // J. Mol. Struct. (Theochem). 1996. V. 388. P. 339.

[11] Jensen F. // J. Chem. Phys. 1999. V. 110. P. 6601.

[12] Dahle P., Helgaker T., Jonsson D., Taylor P.R. // Phys. Chem. Chem. Phys. 2007. V. 9. P. 3112.

[13] Hylleraas E.A. // Z. Phys. 1929. V. 54. P. 29.

[14] James H.M., Coolidge A.S. // J. Chem. Phys. 1933. V. 1. P. 825.

[15] Kolos W., Wolniewicz L. // J. Chem. Phys. 1965. V. 43. P. 2429.

[16] Preiskorn A., Woznicki W. // Mol. Phys. 1984. V. 52. P. 1291.

[17] Cencek W., Rychlewski J. // J. Chem. Phys. 1994. V. 98. P. 1252.

[18] Móller C., Plesset M.S. // Phys. Rev. 1934. V. 46. P. 618.

[19] Klopper W., Manby F.R., Ten-No S., Valeev E.F. // Int. Rev. Phys. Chem. 2006. V. 25. P. 427.

[20] Battaglia S., Bouet D., Lecoq A., Evangelisti S., FaginasLago N. // Mol. Phys. 2020. V. 118. P. e1615646.

[21] Karabulut H. // J. Phys. B: At. Mol. Opt. Phys. 2004. V. 37. P. 3103.

[22] Hamilton I.P., Light J.C. // J. Chem. Phys. 1986. V. 84. P. 306.

[23] Sadlej A. // Chem. Phys. Lett. 1977. V. 47. P. 50.

[24] Moncrieff D., Wilson S. // Mol. Phys. 1993. V. 80. P. 461. 
[25] Wilson S. // Int. J. Quant. Chem. 1996. V. 66. P. 47.

[26] Moncrieff D., Wilson S. // J. Phys. B: At. Mol. Opt. Phys. 1993. V. 26. P. 1605.

[27] Glushkov V.N., Wilson S. // Int. J. Quant. Chem. 2002. V. 89. P. 237.

[28] Glushkov V.N., Wilson S. // Int. J. Quant. Chem. 2004. V. 99. P. 903.

[29] Mackarewicz J., Glushkov V.N. // Int. J. Quant.Chem. 2005. V. 102. P. 353.

[30] Глушков В.Н. // Опт. и спектр. 2006. Т. 100. С. 877.

[31] Moncrieff D., Wilson S. // Adv. Quant. Chem. 1999. V. 31. P. 157.

[32] Wilson S. Electron correlation in molecules. Oxford: Clarendon Press, 1984

[33] Glushkov V.N. // Chem. Phys. Lett. 1998. V. 287. P. 189.

[34] Glushkov V.N., Assfeld X. // Theor. Chem. Acc. 2016. V. 135. P. 3.

[35] Glushkov V.N., Assfeld X. // J. Comp. Chem. 2012. V. 33. P. 2058.

[36] Gilbert A.T.B., Besly N.A., Gill P.M.W. // J. Chem. Phys. 2008. V. A312. P. 13164.

[37] Glushkov V.N. //J. Math. Chem. 2002. V. 31. P. 91.

[38] Glushkov V.N., Assfeld X. // J. Mol. Modeling. 2019. V. 25. P. 148.

[39] Baxter C.A., Cook D.B. // Electronic J. Theor. Chem. 1997. V. 2. P. 66.

[40] Glushkov V.N., Kobus J., Wilson S. // J. Phys. B: At. Mol. Opt. Phys. 2008. V. 41. P. 205102.

[41] Theodorakopoulos G., Petsalakis I.D., Nicolaides C.A., Buenker R.J. // J. Phys.: At. Mol. Phys. B. 1987. V. 20. P. 2339.

[42] Petsalakis I.D., Theodorakopoulos G., Nicolaides C.A., Buenker R.J. // J. Phys.: At. Mol. Phys. B. 1987. V. 20. P. 5959. 